\title{
Transient Common Peroneal and Tibial Nerve Palsy Following Knee Arthroscopy for the Treatment of Discoid Lateral Meniscus
}

\author{
Gabriele Bernardi $^{1}$ Cosimo Tudisco ${ }^{1}$ \\ ${ }^{1}$ Department of Orthopedic Surgery, University of Rome Tor Vergata, \\ Rome, Italy \\ Address for correspondence Gabriele Bernardi, MD, Department of \\ Orthopedic Surgery, University of Rome Tor Vergata, Viale Oxford 81, \\ 00133 Rome, Italy (e-mail: bernardi.gab@gmail.com). \\ Joints 2017;5:118-120.
}

\begin{abstract}
Keywords

- complications

- knee arthroscopy

- tibial nerve

- common peroneal nerve

- discoid meniscus

The number of knee arthroscopies has increased rapidly since the 1980s and are among the most common orthopedic procedures today. It is generally considered a minimally invasive surgery with relatively low morbidity, but it is not without risk of complications. We report the case of a 14-year-old boy who developed a lesion of common peroneal nerve and tibial nerve after knee arthroscopy for a torn discoid lateral meniscus, which was confirmed by electromyogram study.
\end{abstract}

\section{Introduction}

The use of arthroscopic knee surgery has increased significantly since 1980, and arthroscopic meniscectomy is the most commonly performed orthopedic surgery in the United States. ${ }^{1}$ The number of arthroscopic knee procedures continues to increase, because it has reduced the duration of hospitalization with the related costs, broader indications, and has reduced time required for the patient to return to work or sports activities.

Although arthroscopy appears to be a relatively safe procedure, with low invasiveness, and low morbidity, is not free of complications. Several retrospective studies with large series reported overall complication rates ranging between 0.6 and $8.2 \%{ }^{2-4}$

Neurovascular complications are rare but potentially disabling and range from 0.01 to $0.06 \%{ }^{2}$

The nerves most frequently involved are the saphenous nerve and the common peroneal nerve and to a lesser extent the femoral, tibial, and sciatic nerves.

Vascular complications, which may be due to penetration or laceration of the vessels at the time of surgery ${ }^{2,3}$ or secondary to compartment syndrome caused by excessive fluid extravasation due to capsular defects, ${ }^{3}$ are very rare with an estimated incidence $0.003 \%{ }^{2}$

We present a case of common peroneal and tibial nerve palsy after knee arthroscopy for the treatment of a lateral discoid meniscus, which was not directly damaged at the time of surgery.

\section{Case Report}

A 14-year-old boy sustained a twisting injury to his left knee while playing football. A week after the trauma, he visited our department of sports traumatology: the knee appeared moderately swollen, slightly flexed, and the patient complained pain on the lateral compartment during walking, running, and flexion of the knee. Physical examination revealed no ligamentous instability and a full range of motion. The McMurray test resulted in pain along the lateral joint line. No sensory or motor deficits were noted after the injury. Magnetic resonance imaging showed a torn discoid lateral meniscus.

Surgery was performed a week later, with intra-articular anesthesia (20 mL of $1.5 \%$ mepivacaine) and light sedation (propofol $2 \%, 3 \mathrm{mg} / \mathrm{kg} / \mathrm{h}$ ) without the use of a pneumatic
DOI https://doi.org/ $10.1055 / \mathrm{s}-0037-1603674$ ISSN 2282-4324.
Copyright @ 2017 Georg Thieme Verlag KG Stuttgart · New York

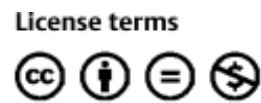



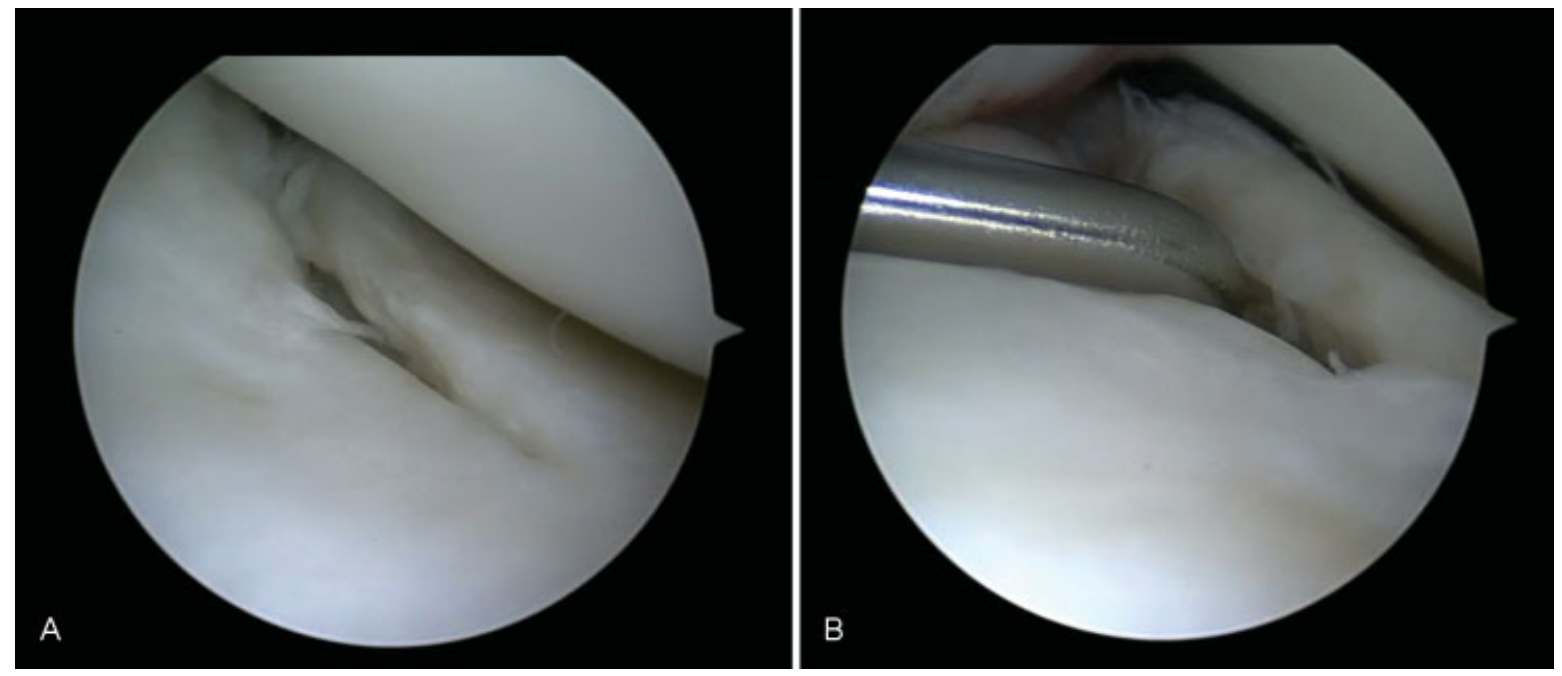

Fig. 1 Torn lateral discoid meniscus. (A) Arthroscopic view from the anterolateral portal. (B) A probe is palpating the tear.

tourniquet. Arthroscopy showed a torn discoid lateral meniscus (type I according to Watanabe's classification) (-Fig. 1A, B).

Anterolateral and anteromedial portals were used with the knee placed in the figure-of-four position, without external compression, and moderate varus stress was applied just raising the foot off the operative table and the contralateral leg. A two pieces excision was done using a monopolar ablator (CoolCut Ablator Hook 90 degrees; Arthrex, Naples, Florida, United States), with a total duration of the procedure of 20 minutes.

Postoperatively the patient noted the foot drop, the inability to flex and extend the ankle and the toes, with an associated sensory loss on the back of the foot and hypoesthesia on the calf and the plant of the foot.

The following day he was discharged with a diagnosis of neurapraxia of tibial and common peroneal nerves and a period of clinical observation was recommended.

After 15 days of the procedure, a physical examination revealed the persistence of inability to flex and extend the ankle and the hallux, associated with a sensory deficit to the foot. Due to the persistence of symptoms, the patient underwent electromyogram study, which showed neurapraxia of tibial and common peroneal nerves. According to the neurologist and neurosurgeon, by virtue of a young age, it was decided to continue conservative pharmacologic treatment (betamethasone $4 \mathrm{mg}$ injection twice a day for 3 days, followed by $4 \mathrm{mg}$ daily for 4 days), physical therapy, foot drop splint, and clinical reassessment 15 days later.

After 15 days of observation, clinical examination showed restoration of sensitivity to the plant of the foot, hypoesthesia of the dorsum of the foot, muscle strength (according to the British Medical Research Council grading, BMRC) 4/5 of the gastrocnemius-soleus complex and $3 / 5$ of the tibialis anterior, extensor hallucis longus, and peroneal muscles.

After 2 months of the surgery, the clinical examination showed the persistence of the hypoesthesia of the dorsum of the foot and complete recovery of motor function.

\section{Discussion}

Most of the information regarding complications after knee arthroscopy dates back to the 1980s, with rates ranging between 0.6 and $8.2 \%{ }^{2-4} \mathrm{~A}$ review of the literature found few recent reports ${ }^{5,6}$ showing a decrease in overall complications rates that may be due to advances in equipment, the evolution of techniques, and increased familiarity of the surgeons with arthroscopic procedures.

Sherman et $\mathrm{al}^{3}$ retrospectively reviewed 2,640 arthroscopies performed by four surgeons: there were 216 complications (overall 8.2\%; nervous complications were $0.6 \%$ ).

DeLee $^{4}$ reported the incidence of nerve complications of $0.05 \%$ on 118,850 arthroscopies, while Small ${ }^{2}$ reported a neurological complication rate of $0.06 \%$ on 375,069 arthroscopic knee procedures. Subsequently, Small ${ }^{7}$ reported a prospective study in which 10,262 procedures were registered, and reported an overall complication rate of $1.68 \%$. The most common complications in that study were haemarthrosis (60.1\%), infection (12.1\%), thromboembolic disease (6.9\%), anesthetic complications (6.4\%), instrument failure $(2.9 \%)$, complex regional pain syndrome $(2.3 \%)$, ligament injury (1.2\%), and fracture or neurological injury (0.6\% each).

Most reports regarding neurological lesions involved neurapraxia of the saphenous nerve (in particular the infrapatellar branch) due to direct damage from the trocar of the anteromedial portal and lesions of the peroneal nerve due to various mechanisms that include the entrapment during inside-out meniscal sutures, the positioning of the posterolateral portal or violation of posterolateral corner.

The newest reports on the complications of arthroscopic knee surgery reported an overall complication rate between 0.27 and $4.7 \% .^{6,8,9}$

To the best of our knowledge, there is just one report in the literature $^{10}$ of an iatrogenic lesion of the common peroneal and tibial nerves caused by arthroscopy that was caused by violation of the posterolateral corner by powered 
arthroscopic instrumentation during the attempt to remove an osteochondral loose body.

In our case, the tibial and peroneal nerves palsy occurred in the absence of direct trauma during surgery, in the absence of tourniquet or excessive valgus stress. We believe that the nerve damage was caused by nervous traction related patient positioning during surgery; our advice is to bring back the knee to a neutral position (either in full extension on the operative table, or hanging from a leg holder) at regular intervals (e.g., every 10 minutes or more frequently) to relax the neurovascular structures.

There is only one case, as described by Johnson et al ${ }^{11}$ of peroneal nerve palsy, probably due to a nerve stretching injury, with partial motor recovery, and no sensory recovery 17 months after arthroscopic meniscectomy in a 43-year-old woman.

Most of neurological lower extremity injuries due to surgical position are described in gynecological literature because of prolonged use of the lithotomy position, a position in which the patients lie on their back with the hips and knees flexed and the thighs apart, which can lead to a traction injury of sciatic nerve, probably due to microvascular compromise or local mechanical compression of the nerve.

\section{Conclusion}

Despite the widespread use of knee arthroscopy, the rate of overall complications is usually low, and patients and surgeons should be aware of the possibility of nerve damage. Furthermore, the proper patient positioning is very important during the procedure, to prevent nerve injury that can impair a successful surgical procedure, be highly disabling for the patient, as well as expose the surgeon to possible medicolegal conflict.

\section{References}

1 Garrett WE Jr, Swiontkowski MF, Weinstein JN, et al. American Board of Orthopaedic Surgery Practice of the Orthopaedic Surgeon: part-II, certification examination case mix. J Bone Joint Surg Am 2006;88(03):660-667

2 Small NC; Committee on Complications of the Arthroscopy Association of North America. Complications in arthroscopy: the knee and other joints. Arthroscopy 1986;2(04):253-258

3 Sherman OH, Fox JM, Snyder SJ, et al. Arthroscopy-"no-problem surgery." An analysis of complications in two thousand six hundred and forty cases. J Bone Joint Surg Am 1986;68(02): 256-265

4 DeLee JC; Committee on Complications of Arthroscopy Association of North America. Complications of arthroscopy and arthroscopic surgery: results of a national survey. Arthroscopy 1985; 1(04):214-220

5 Jameson SS, Dowen D, James P, Serrano-Pedraza I, Reed MR, Deehan DJ. The burden of arthroscopy of the knee: a contemporary analysis of data from the English NHS. J Bone Joint Surg $\mathrm{Br}$ 2011;93(10):1327-1333(Br Vol)

6 Salzler MJ, Lin A, Miller CD, Herold S, Irrgang JJ, Harner CD. Complications after arthroscopic knee surgery. Am J Sports Med 2014;42(02):292-296

7 Small NC. Complications in arthroscopic surgery performed by experienced arthroscopists. Arthroscopy 1988;4(03):215-221

8 Martin CT, Pugely AJ, Gao Y, Wolf BR. Risk factors for thirty-day morbidity and mortality following knee arthroscopy: a review of 12,271 patients from the national surgical quality improvement program database. J Bone Joint Surg Am 2013;95(14):e98, 1-10

9 Hagino T, Ochiai S, Watanabe Y, et al. Complications after arthroscopic knee surgery. Arch Orthop Trauma Surg 2014;134(11): 1561-1564

10 Wendt MC, Spinner RJ, Shin AY. Iatrogenic transection of the peroneal and partial transection of the tibial nerve during arthroscopic lateral meniscal debridement and removal of osteochondral fragment. Am J Orthop 2014;43(04):182-185

11 Johnson DS, Sharma DP, Bangash IH. Common peroneal nerve palsy following knee arthroscopy. Arthroscopy 1999;15(07): 773-774 